\section{Cureus}

Received 08/12/2019

Review began 08/14/2019

Review ended 08/18/2019

Published 08/19/2019

\section{(C) Copyright 2019}

Lin et al. This is an open access article distributed under the terms of the Creative Commons Attribution License CC-BY 3.0., which permits unrestricted use, distribution, and reproduction in any medium, provided the original author and source are credited.

\title{
Intranasal Acetaminophen Abuse and Nasal, Pharyngeal, and Laryngotracheal Damage
}

Yufan Lin ${ }^{1}$, Jennifer Y. Lu ${ }^{1}$, Carlos D. Pinheiro-Neto ${ }^{1}$, David M. Jones ${ }^{2}$, Neil GildenerLeapman $^{1}$

1. Department of Surgery, Division of Otolaryngology, Albany Medical Center, Albany, USA 2. Department of Pathology, Albany Medical Center, Albany, USA

$\square$ Corresponding author: Neil Gildener-Leapman, gildenn2@amc.edu Disclosures can be found in Additional Information at the end of the article

\section{Abstract}

A young adult female originally presented with necrosis of the nasal cavity mucosa and septum after sniffing crushed acetaminophen. She underwent endoscopic sinus surgery and debridement but continued to use acetaminophen intranasally. Four months later, the destruction had extended to include the posterior pharyngeal wall and subglottis. The diagnosis was confirmed by polarizable talc found on biopsy of the subglottis. While nasal insufflation of cocaine and hydrocodone-acetaminophen has been well-documented, intranasal abuse of exclusively acetaminophen is not well understood. This case demonstrates the destructive potential of intranasal acetaminophen use and may help physicians recognize unusual signs and symptoms of intranasal drug abuse.

Categories: Otolaryngology, Pathology

Keywords: acetaminophen, intranasal drug abuse, polarizable talc, septum perforation, subglottic ulceration

\section{Introduction}

Nasal insufflation has long been associated with abuse of cocaine and more recently, prescription narcotics. The most commonly prescribed form of prescription narcotic abuse is in the form of hydrocodone-acetaminophen [1]. When these medications are abused via nasal insufflation, there is a well-documented history of necrosis of the nasal septum, soft palate, and hard palate. These patients often present with nasal pain, septal perforations, and noninvasive fungal infection [2]. This report describes a case of intranasal abuse of exclusively acetaminophen leading to damage from the nose down to the subglottis. Intranasal abuse of acetaminophen alone is not well-documented and this case may suggest a new trend in drug abuse. It has been seen in only one case study prior despite growing in popularity in the community [3].

\section{Case Presentation}

A young adult female with a history of chronic pain, multi-substance abuse, and obsessivecompulsive disorder originally presented with a history of necrosis of her nasal septum with chronic crusting for greater than six months. She also reported symptoms consistent with Eustachian tube dysfunction, but no hearing loss. At that time, she reported that she was only sniffing crushed over-the-counter acetaminophen. Additionally, she takes a serotonin reuptake inhibitor, but reports she does not snort that. On nasal endoscopy, there was near-complete 


\section{Cureus}

destruction of the nasal septum with crusting of white powder and secretions in the nasal cavity (Figure 1). A computed tomography (CT) scan of her sinuses demonstrated septal perforation as well as pansinusitis with a mucosal thickening.

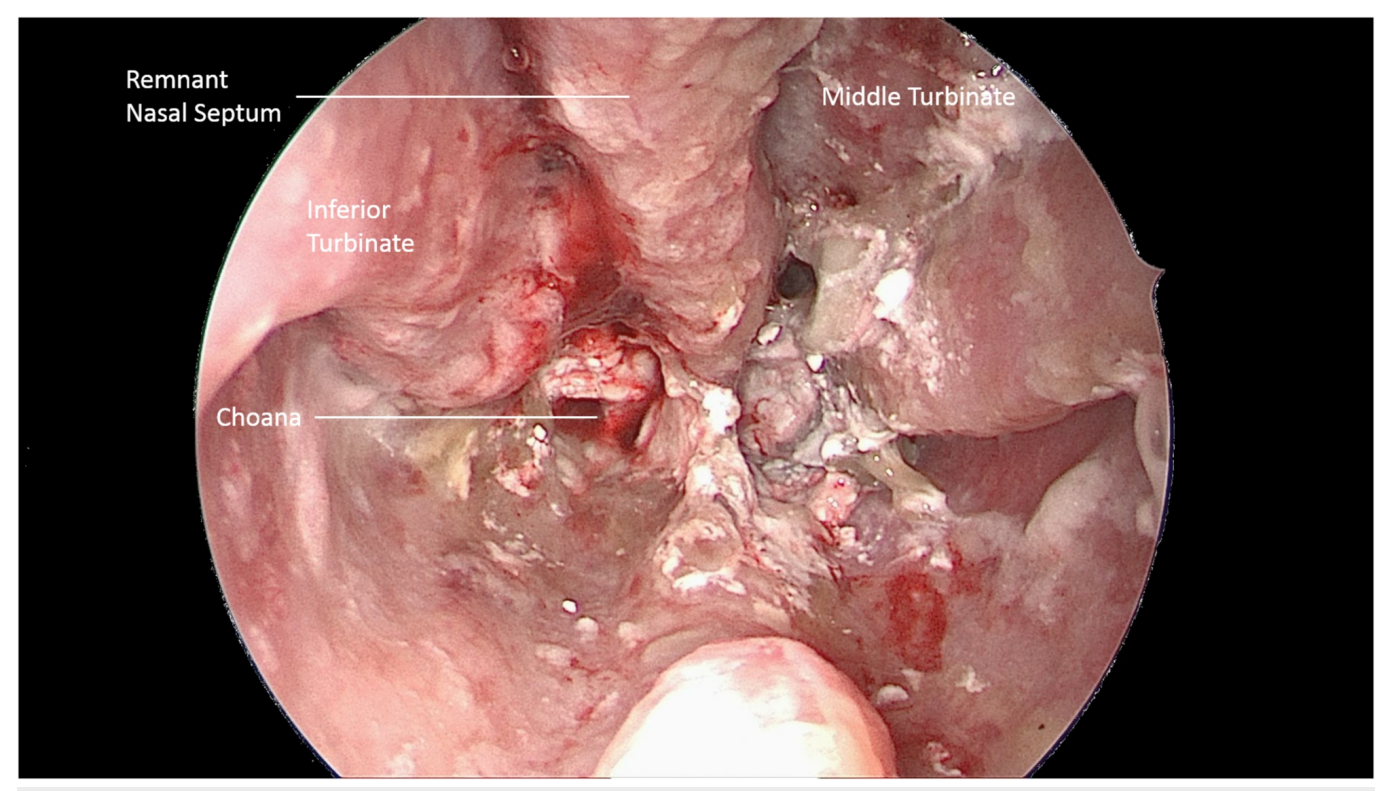

\section{FIGURE 1: Endoscopic Evidence of Damage}

Nasal endoscopy obtained with a 0-degree endoscope demonstrating near total septectomy, pill residue, and debris. Diffuse erosive damage was observed along the nasal mucosa with crusted pill debris and blood occluding the choanae and middle meatus.

At this time, the patient was taken to the operating room for endoscopic sinus surgery including bilateral maxillary antrostomy, total ethmoidectomies, and sphenoidotomies. Hematoxylin and eosin stained sections of a nasal mucosal biopsy reveal ulcerated mucosa with attached fibrinopurulent debris and refractile foreign material. Intact portions of mucosa reveal a lichenoid host response and focal subepithelial sclerosis (Figure 2). Her postoperative course was unremarkable, however she continued to use acetaminophen intranasally and was unable to abstain. 


\section{Cureus}

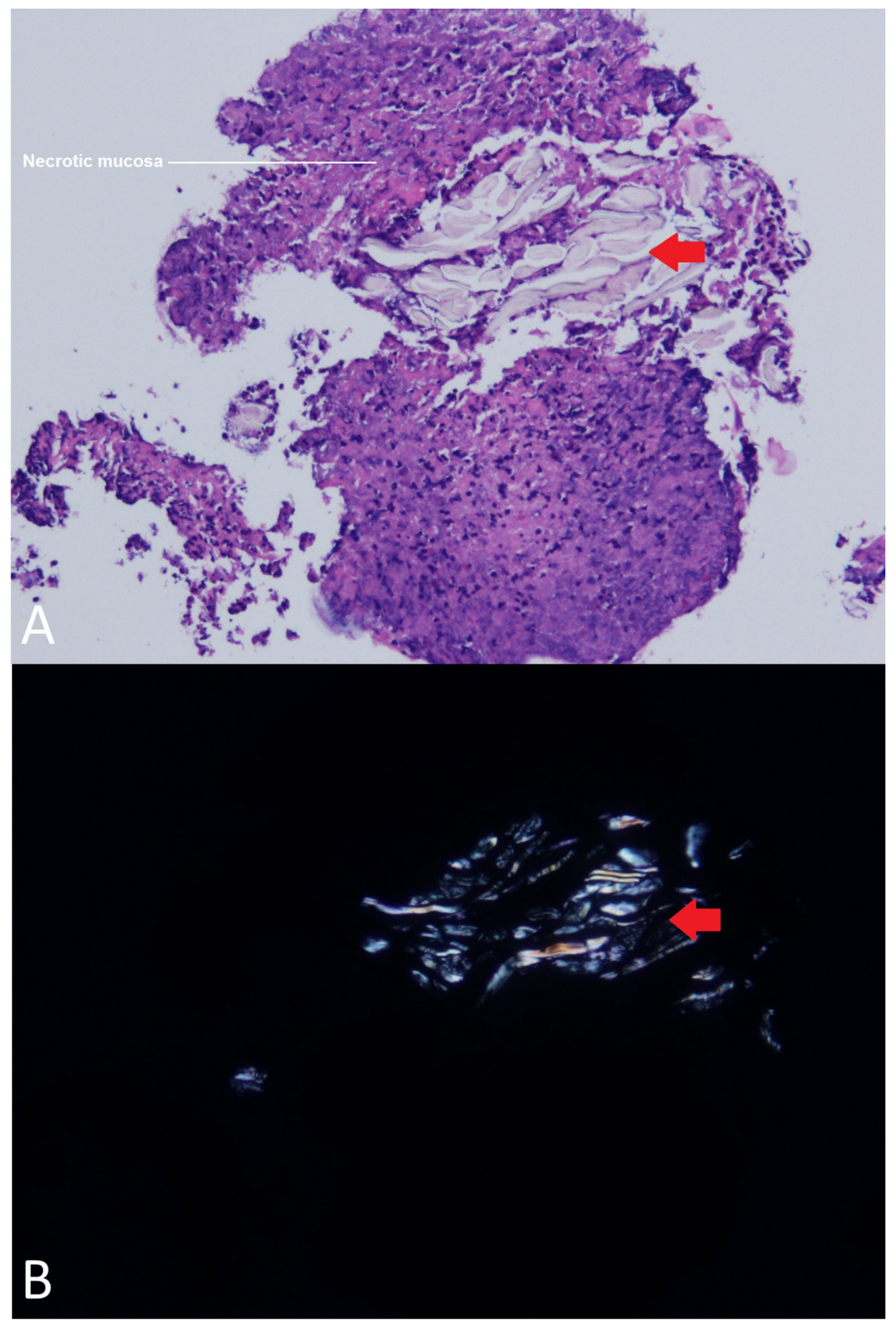

\section{FIGURE 2: Intranasal Pill Particles on Ulcerated Mucosa on}

\section{Hematoxylin and Eosin}

A) 100x magnification of hematoxylin and eosin stained slide showing ulcerated mucosa (white line) and refractile pill material (red arrowhead); B) 100x magnification slide with polarized light highlighting talc fragments from pill remnants. 


\section{Cureus}

She presented to the office four months later with continued postnasal drip, nasal crusting, and eustachian tube dysfunction with a normal audiogram. She also had new complaints of intense pharyngeal pain that interrupted her sleep and periodic hoarseness without dyspnea. She reported a 20 pound weight loss over the past year, with a current body mass index (BMI) of 15 . On exam, her voice was hoarse and asthenic. On flexible nasolaryngoscopy, she had recurrent crusting in the nasal cavity and the mucosa was not visible. The nasopharynx and the posterior pharyngeal wall had erosive yellow eschar and there was an anterior subglottic lesion. Important laboratory values include: elevated c-reactive protein at $5.30 \mathrm{mg} / \mathrm{L}$ (reference range $<3.00 \mathrm{mg} / \mathrm{L}$ ) and erythrocyte sedimentation rate was elevated at $33 \mathrm{~mm} / \mathrm{h}$ (reference range 0-20 $\mathrm{mm} / \mathrm{h}$ ). Her white blood cell count was $10.7 \times 10^{9}$ cells/L (reference range 4.0-10.0 x10 ${ }^{9}$ cells/L), with elevated absolute neutrophil count at $6.47 \times 10^{9}$ cells/L (reference range $1.5-6 \times 10^{9}$ cells/L). Her anti-nuclear antibody titer, rheumatoid factor, perinuclear anti-neutrophil cytoplasmic antibody titer, and cytoplasmic anti-neutrophil cytoplasmic antibody titer were all negative.

The patient was educated on nasal humidification, including nasal saline irrigation, as well as intranasal petroleum jelly. In several weeks, despite continued acetaminophen use, her nasal cavity was successfully debrided and normal mucosa was seen throughout the nose. The patient was taken to the operating room for direct laryngoscopy and biopsy (Figure 3). In the operating room, the findings of posterior pharyngeal wall ulceration and subglottic soft tissue lesion were confirmed. Biopsies taken of the posterior pharyngeal wall and subglottis showed a nonulcerated squamous mucosa with an inflammatory infiltrate and refractile foreign material.

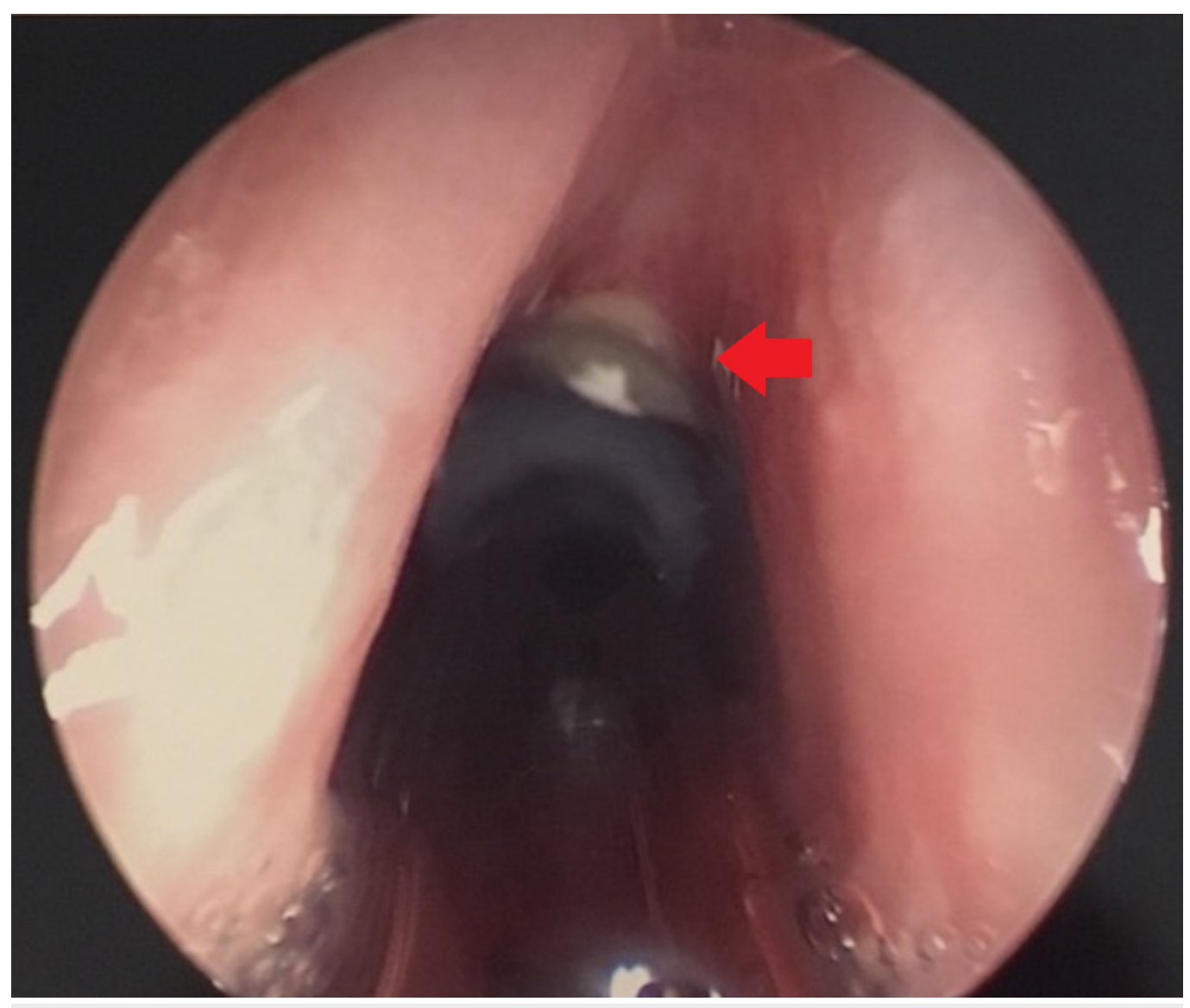

\section{FIGURE 3: View on Direct Laryngoscopy}

Anterior subglottic ulcerated tissue and debris (arrowhead). 
The patient enrolled in a drug abstinence program for several weeks, which allowed for healing of the ulcerated lesions, resolution of pharyngeal pain, and voice complaints. Unfortunately, the patient restarted intranasal acetaminophen use and requires regular in-office nasal debridement, and continues to have some pharyngeal discomfort as well as vocal strain.

\section{Discussion}

Intranasal abuse of exclusively acetaminophen is not well described in the scientific literature. Studies of intranasal abuse of acetaminophen almost always examine crushed tablets of acetaminophen in combination with another drug, usually hydrocodone. Hardison et al. described a case of exclusively acetaminophen in a 2015 case study of a 34-year-old male, but no other cases can be found [3]. In addition to being a rare documented case of acetaminophen abuse, this case report is unique in the extent of damage, with necrosis and talc expanding into the subglottic region.

The key to recognition of this entity is pathologist and clinician communication about the identification of both non-polarizable and polarizable foreign talc fragments. Talc is used as a tablet binder for acetaminophen and other oral medications; it appears as birefringent, needleshaped particles under polarized light [4]. Originally, the differential diagnosis for this patient included various disorders causing subglottic inflammation. Autoimmune diseases such as granulomatosis with polyangiitis with sinus and subglottic involvement were considered [5]. Talc on histological finding and negative anti-neutrophil cytoplasmic antibody (ANCA) panel differentiate intranasal drug abuse from the other possible immune etiologies [2].

Acetaminophen is especially appealing because this medication is much easier to obtain, lacking the legal substance controls that regulate cocaine and hydrocodone-acetaminophen. However, whereas the motivation to abuse intranasal opiates is better characterized, abuse of intranasal acetaminophen alone is not well understood. Psychotropic effects including sensations of calm and general feelings of pleasantness have been documented in individual patient experience reports [6]. Pharmacodynamic studies of combined intranasal opiateacetaminophen use demonstrate faster onset to maximum serum concentrations compared to oral administration as well as slightly elevated peak concentrations [7], which may increase the abuse potential of intranasal use. It is uncertain if our patient derived a high degree of euphoria from her acetaminophen use as she lacked insight about her behavior. We can only speculate about the degree to which her addiction was influenced by perceived benefit versus physiologic effect.

As this method of drug abuse gains popularity, medical professions can expect to see an increase in nasopharyngeal mucosal damage. The physician should also consider potential for hepatotoxicity with acetaminophen used in excess. Long-term follow up of these patients is helpful because abstinence, in this case, was not easy to maintain.

\section{Conclusions}

Even in the absence of prescription and recreational drugs, insufflation should be considered in the differential for nasopharyngeal necrosis. This case report illustrates the intersection of mental health issues and substance abuse involving an easily accessible over-the-counter medication. As this pattern of abuse gains popularity, physicians need to maintain a high index of suspicion for intranasal medication abuse.

\section{Additional Information \\ Disclosures}


Human subjects: Consent was obtained by all participants in this study. Conflicts of interest: In compliance with the ICMJE uniform disclosure form, all authors declare the following:

Payment/services info: All authors have declared that no financial support was received from any organization for the submitted work. Financial relationships: All authors have declared that they have no financial relationships at present or within the previous three years with any organizations that might have an interest in the submitted work. Other relationships: All authors have declared that there are no other relationships or activities that could appear to have influenced the submitted work.

\section{References}

1. Alexander D, Alexander K, Valentino J: Intranasal hydrocodone-acetaminophen abuse induced necrosis of the nasal cavity and pharynx. Laryngoscope. 2012, 122:2378-2381. 10.1002/lary.23542

2. Vosler PS, Ferguson BJ, Contreras JI, Wang EW, Schaitkin BM, Lee S: Clinical and pathologic characteristics of intranasal abuse of combined opioid-acetaminophen medications. Int Forum Allergy Rhinol. 2014, 4:839-844. 10.1002/alr.21355

3. Hardison SA, Marcum KK, Lintzenich CR: Severe necrosis of the palate and nasal septum resulting from intranasal abuse of acetaminophen. Ear Nose Throat J. 2015, 94:40-42.

4. Marchiori E, Lourenco S, Gasparetto TD, Zanetti G, Mano CM, Nobre LF: Pulmonary talcosis: imaging findings. Lung. 2010, 188:165-171. 10.1007/s00408-010-9230-y

5. Al-Qadi MO, Artenstein AW, Braman SS: The "forgotten zone": acquired disorders of the trachea in adults. Respir Med. 2013, 107:1301-1313. 10.1016/j.rmed.2013.03.017

6. Abbott FV, Hellemans KGC: Phenacetin, acetaminophen and dipyrone: analgesic and rewarding effects. Behav Brain Res. 2000, 112:177-186. 10.1016/s0166-4328(00)00179-0

7. Guenther SM, Mickle TC, Barrett AC, Roupe KA, Zhou J, Lam V: Relative bioavailability, intranasal abuse potential, and safety of benzhydrocodone/acetaminophen compared with hydrocodone bitartrate/acetaminophen in recreational drug abusers. Pain Med. 2018, 19:955966. $10.1093 / \mathrm{pm} / \mathrm{pnx} 195$ 